\title{
THE HARDWARE IMPLEMENTATION OF THE MULTI-POSITION SIGNAL DIGITAL DEMODULATORS
}

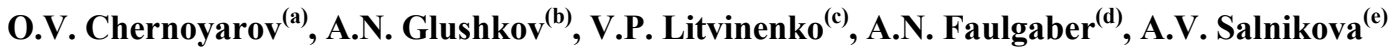 \\ (a),(d),(e) National Research University "Moscow Power Engineering Institute", Moscow, Russia

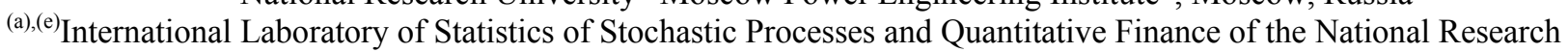 \\ Tomsk State University, Tomsk, Russia

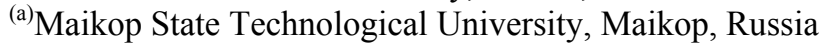

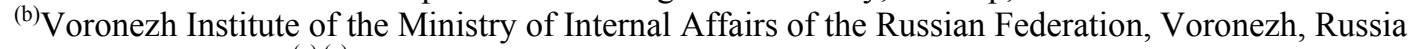 \\ ${ }^{(\mathrm{c}),(\mathrm{e})}$ Voronezh State Technical University, Voronezh, Russia \\ ${ }^{(a)}$ chernoyarovov@mpei.ru, ${ }^{(b)}$ a.n.glushkov@,gmail.com, ${ }^{(c)}$ v.p.litvinenko@gmail.com ${ }^{(c)}$ lytaleksandr@mail.ru \\ (d) amicus.lat@yandex.ru
}

\begin{abstract}
There are considered the capabilities of the hardware implementation of the digital demodulators when receiving the signals of various modulation formats. It is shown that the multi-position signal processing devices can be implemented by means of the on the relatively inexpensive field programmable gate arrays.
\end{abstract}

Keywords: workstation design, work measurement, ergonomics, decision support system

\section{INTRODUCTION}

The digital modulation signals (PSK, QAM, etc.) are widely used in radio communication systems (Feher, 1995; Sklar, 2001; Proakis and Salehi 2007). The application of modern equipment is focused on the digital implementation of phase detectors and demodulators. Hence, in the present paper, devices are introduced requiring performing only a minimum number of simple arithmetic operations over the signal period (Chernoyarov et al. 2015-2018). Yet, software implementation of the algorithms for processing highfrequency digital modulation signals in real time when it is carried out by means of the signal processors requires too significant computational costs. In view of this, the field programmable gate arrays (FPGAs) are considered as more appropriate tools for hardware processing of the high-frequency signals in terms of the specified algorithms. Below, the resources are analyzed that are required for the implementation of the demodulators by means of the FPGAs.

\section{THE MAJOR PART}

An urgent task in designing the digital demodulators is to estimate hardware resources needed for their FPGAbased implementation. The most resource-intensive receiving devices are the quadrature amplitude modulation (QAM) signal demodulator (Chernoyarov, Glushkov, Litvinenko, Litvinenko and Matveev 2018) and the non-coherent demodulator of the signals phaseshift keyed in toto (Glushkov, Litvinenko, Matveev,
Chenoyarov and Kalashnikov 2016; Chernoyarov, Litvinenko, Glushkov, Matveev and Salnikova 2017). Modern FPGAs (Andina, de la Torre Arnanz and Valdes 2017) provide greater opportunities for the implementation of the digital high-frequency signal demodulators, but in this case, however, there should be designed the processing units requiring minimal computational resources.

In common studies (Chernoyarov O.V. et al. 20152018), the basic fast digital algorithms for coherent and noncoherent signal processing are introduced. In Fig. 1, the block diagram of the noncoherent processing algorithm is shown.

The signal from the intermediate frequency section output of the receiver is fed to the input of the 8-to 16bit analog-to-digital converter (ADC) operated by the clock generator (CG) with the frequency $f_{Q}=4 f_{0}$.

Here $f_{0}$ is the band center of the signal that may have frequency of tens of megahertz. As a result, the four samples $s_{1 i}, s_{i 1}, s_{i 2}, s_{i 3}$ are formed over each of the $i$ th signal period $T_{0}=1 / f_{0}$ as shown in Fig. 2. They are stored in the multi-bit four-cell shifter (MS4), while in the subtractors $\mathrm{SUB}_{0}$ and $\mathrm{SUB}_{1}$ the differences are determined of the even and odd samples forming the quadrature processing channels of the signal.

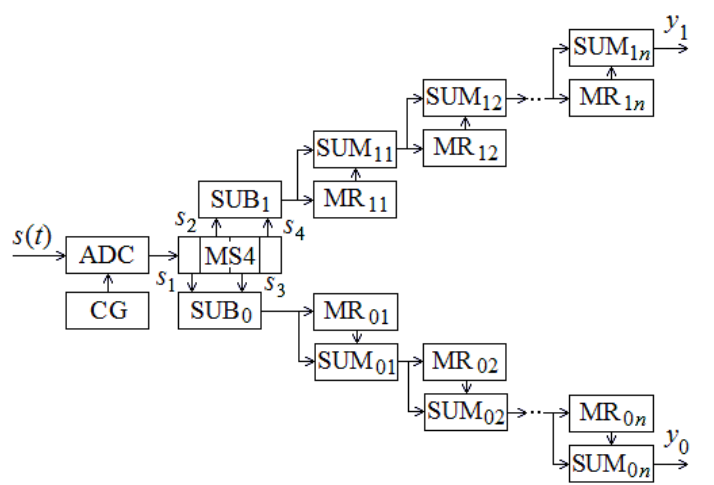

Figure 1: The block diagram of the noncoherent processing algorithm 


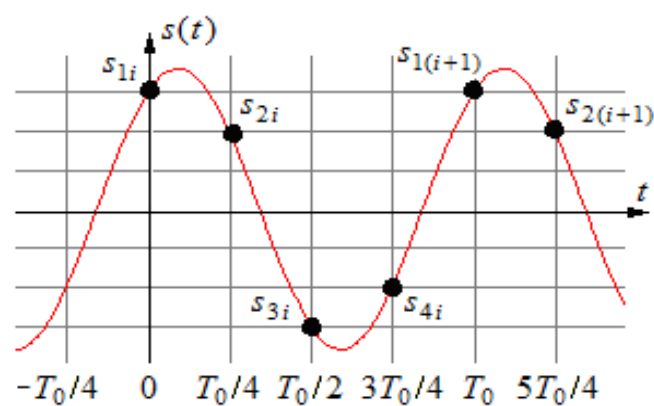

Figure 2: Time quantization diagram

The pairs of even and odd current and previous (stored into multi-bit shift registers $\mathrm{MR}_{01}$ and $\mathrm{MR}_{11}$ ) differences of the samples are summarized in the summators $\mathrm{SUM}_{01}$ and $\mathrm{SUM}_{11}$. Further, the obtained sums (two, then four, then eight, etc.) are accumulated in the subsequent summators, so that the responses of the quadrature channels are formed over the interval of the information signal duration $N=2^{n}$ (where $n$ is an integer) as follows

$$
y_{0 i}=\sum_{j=0}^{N-1}\left(s_{1(i-j)}-s_{3(i-j)}\right), y_{1 i}=\sum_{j=0}^{N-1}\left(s_{2(i-j)}-s_{4(i-j)}\right) .
$$

The basic algorithms provide the minimum number $n=\log _{2} N$ of the addition operations for accumulating the differences of the signal samples for the sample of the size $N$ in each of the quadrature channels (Fig. 1). This also applies to the coherent algorithm (Glushkov, Litvinenko, Matveev, Chernoyarov and Salnikova 2015; Chernoyarov, Glushkov, Litvinenko, Litvinenko and Matveev B.V. 2017).

Based on these basic and coherent algorithms, various devices can be implemented for demodulating highfrequency phase-shift keyed (PSK) and differential phase-shift keyed (DPSK) radio signals.

In Fig. 3a, the block diagram is shown of the digital binary DPSK signal demodulator, while in Fig. 3b we can see the block diagram of the four-position DPSK signal demodulator (Chernoyarov, Glushkov, Litvinenko, Litvinenko and Matveev 2018).

In comparison with the basic algorithm (Fig. 1) in the demodulator circuit presented in Fig. 3a, there are supplemented the multi-bit $N$ cell shifters $\mathrm{MR}_{0(n+1)}$ and $\mathrm{MR}_{1(n+1)}$, two additional summators and subtractors, two quadratic blocks (QT) performing the operations

$$
\begin{aligned}
& z_{0 i}=\left(y_{01 i}+y_{0(i-N)}\right)^{2}+\left(y_{1 i}+y_{1(i-N)}\right)^{2}, \\
& z_{1 i}=\left(y_{01 i}-y_{0(i-N)}\right)^{2}+\left(y_{1 i}-y_{1(i-N)}\right)^{2},
\end{aligned}
$$

as well as the substractor (SUB) and the comparator (C) forming the information symbol $S_{I}$.

Similarly, into the four-position DPSK signal demodulator (Fig. 3b), the basic algorithm is supplemented by the computing unit (CU) that performs the operations

$$
\begin{aligned}
& z_{0}=y_{0, i} y_{0,(i-N)}+y_{1, i} y_{1,(i-N)}+y_{1, i} y_{0,(i-N)}-y_{0, i} y_{1,(i-N)}, \\
& z_{1}=y_{0, i} y_{0,(i-N)}+y_{1, i} y_{1,(i-N)}-y_{1, i} y_{0,(i-N)}+y_{0, i} y_{1,(i-N)},
\end{aligned}
$$

as well as the two comparators $\mathrm{C}_{1}$ and $\mathrm{C}_{2}$ at the outputs of which the symbols $S_{I 0}$ and $S_{I 1}$ are generated. The block diagrams of the coherent PSK and DPSK signal demodulators are much simpler than those shown in Fig. 3.

Thus, the basic digital algorithm allows us to implement various PSK signal demodulators at relatively low computational costs

In Fig. 4a, the block diagram is shown of a more complex coherent quadrature amplitude modulation (QAM) signal demodulator (Chernoyarov, Glushkov, Litvinenko, Litvinenko and Matveev B.V. 2018). Here the responses of the quadrature channels (2) and (3) are passed to the threshold devices $\mathrm{TD}_{0}$ and $\mathrm{TD}_{1}$ as well as to the normalizing and synchronizing device (NSD) providing clock synchronization and the formation of the thresholds for TDs. According to the output TDs signals, in the resolver (RS) the code words are separated by which the binary code of the received multi-position symbol is formed in the decoder (DC).

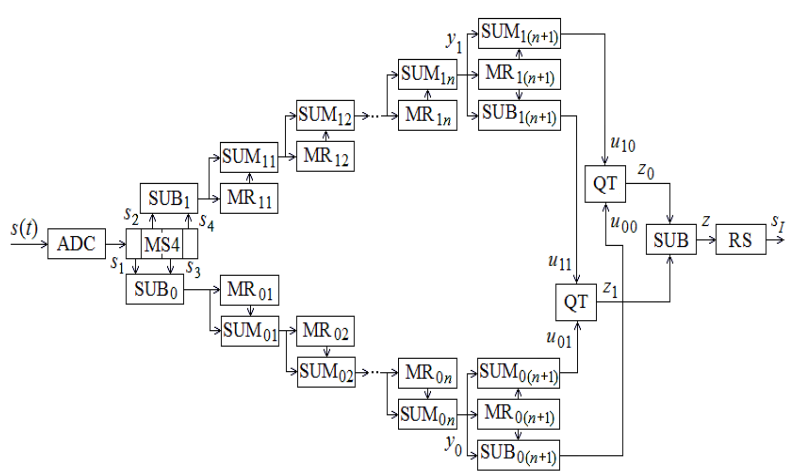

a)

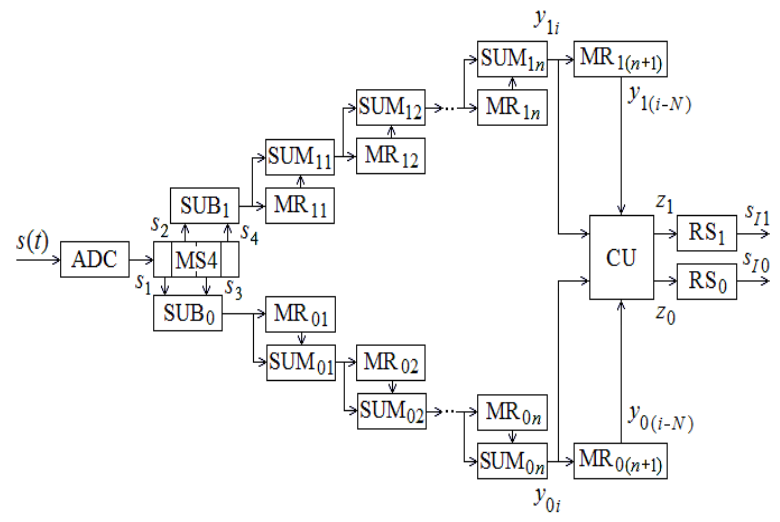

b)

Figure 3: The block diagrams of the fast digital algorithms for noncoherent demodulation of binary (a) and four-position (b) DPSK signals 


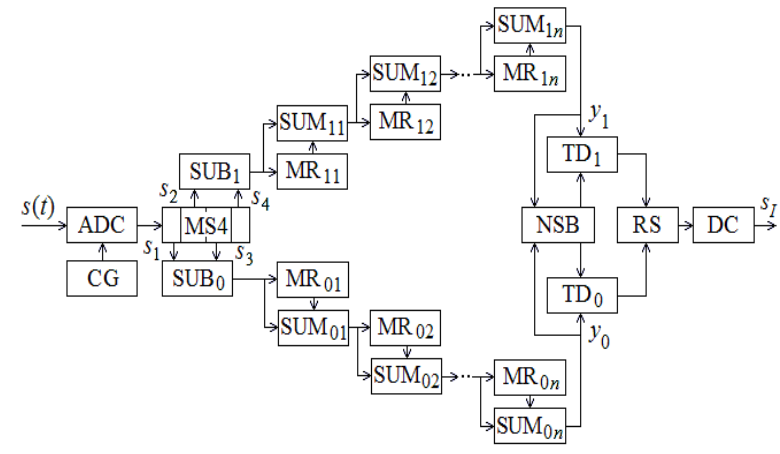

a)

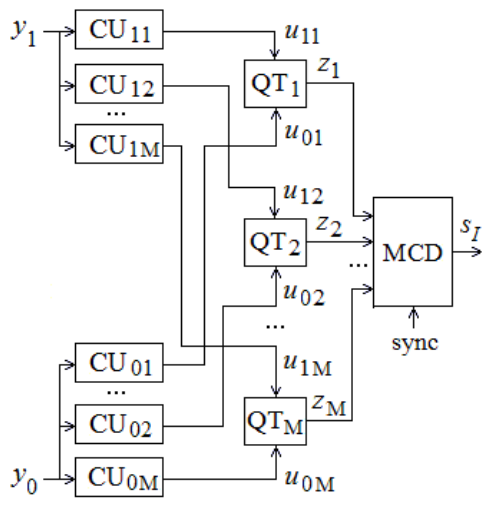

b)

Figure 4: The block diagrams of the fast digital algorithms for noncoherent demodulation of binary (a) and four-position (b) DPSK signals

The digital demodulator of the signals phase-shift keyed in toto (Glushkov, Litvinenko, Matveev, Chenoyarov and Kalashnikov 2016; Chernoyarov, Litvinenko, Glushkov, Matveev and Salnikova 2017) requires the greatest computational cost. The block diagram of its addition to the basic algorithm (Fig. 1) is shown in Fig. 4b.

The values $y_{0 i}, y_{1 i}$ are moved to the computing units $\mathrm{CU}_{0}$ and $\mathrm{CU}_{1}$ generating the responses $u_{0 m}$ and $u_{1 m}$ to the $m$-th code combination of $K$ elements length, where $m=\overline{1, M}$ and $M$ is the number of these code words. For the $i$-th signal period, at the CUs outputs we get

$u_{0 i m}=\sum_{k=0}^{K-1} a_{m k} y_{0(i-k N)}, \quad u_{1 i m}=\sum_{k=0}^{K-1} a_{m k} y_{1(i-k N)}$,

where $a_{m k}= \pm 1$ is the elements of the $m$-th code word. By the transformation

$z_{\text {im }}=\sqrt{u_{0 i m}^{2}+u_{1 i m}^{2}}$.

the demodulator responses are generated for each enabled code combination, and by the largest of these responses a decision on the received code combination $S_{I}$ is made by the maximum choice device (MCD).
To estimate the possibilities of designing the specified demodulators, FPGAs of various modifications have been considered produced by Xilinx (Xilinx DS160 Spartan-6 family overview 2011; 7 Series FPGAs data sheet: Overview 2018) and Altera (Parab J.S., Gad R.S. and Naik G.M. 2018). The selection criteria are the required hardware resources and the cost.

The results of the FPGA resource estimation for implementing the digital binary DPSK signal demodulator (Fig. 3a) are shown in Table 1, for the case when the ADC width is 8 bits and $N=128$. As we can see, a relatively simple demodulation algorithm does not require significant computational power.

In order to implement the demodulator of the signals phase-shift keyed in toto and coded by Walsh sequences (Chernoyarov, Litvinenko, Glushkov, Matveev and Salnikova 2017), FPGAs are used produced by the Xilinx, such as XilinxXC6SL25 (Spartan-6), XC7K70T (Kintex-7), XC7A100T (Artix-7). As an example, for each FPGA, an HDL code has been synthesized and implemented with the fixed parameters: the Walsh code sequence length is $M=4$, the number of carrier periods is $\mathrm{N}=64$, the ADC bus width is $\mathrm{R}=12$. It is established that for the FPGA XC6SL25 the maximum possible carrier signal frequency is $144 \mathrm{MHz}$, while for the $\mathrm{XC7A} 100 \mathrm{~T}$ it is $118 \mathrm{MHz}$ and for the XC7K70T - 154 $\mathrm{MHz}$. The power consumption at the signal frequency of $50 \mathrm{MHz}$ lies within the range from 40 to $90 \mathrm{~mW}$. In Table 2, the results are presented of a quantitative estimation (as percentage of the total) of the required hardware FPGA resources.

In Table 3, the similar results are shown produced while implementing the demodulator of the two signals phase shift keyed in toto and coded by $M$-sequences of $K$ elements length.

As it can be seen, there can be successfully implemented FPGA-based demodulators of the signals with the different digital modulation formats in toto.

Table 1: The estimation of the hardware overhead (per cent) of FPGAs produced by Xilinx for implementing the digital binary DPSK signal demodulator

\begin{tabular}{|c|c|c|c|}
\hline Elements & Spartan 6 & Virtex 5 & Kintex 7 \\
\hline Flip Flops & 5 & $<1 \%$ & $<1 \%$ \\
\hline LUTs & 35 & $2 \%$ & $<1 \%$ \\
\hline Slices & 50 & $3 \%$ & $<1 \%$ \\
\hline DSP & 25 & $6 \%$ & $<1 \%$ \\
\hline
\end{tabular}

Table 2: The estimation of the hardware overhead (per cent) of FPGAs produced by Xilinx for implementing the digital demodulator of the signals phase-shift keyed in toto and coded by Walsh sequences

\begin{tabular}{|c|c|c|c|}
\hline \multirow{2}{*}{ FPGA } & \multicolumn{3}{|c|}{ Elements } \\
\cline { 2 - 4 } & XC6SL25 & XC7K70T & XC7A100T \\
\hline $\begin{array}{c}\text { Slice } \\
\text { Registers }\end{array}$ & 2.55 & 0.93 & 0.6 \\
\hline SliceLUTs & 14.26 & 7.05 & 4.56 \\
\hline DSP & 21.05 & 3.33 & 3.33 \\
\hline
\end{tabular}


Table 3: The estimation of the hardware overhead (per cent) of FPGAs produced by Xilinx for implementing the digital demodulator of the signals phase-shift keyed in toto and coded by $M$-sequences

\begin{tabular}{|c|c|c|c|c|}
\hline \multirow{2}{*}{ FPGA } & \multicolumn{5}{|c|}{ Elements } \\
\cline { 2 - 5 } & $\mathrm{K}$ & XC6SL25 & XC7K70T & XC7A100T \\
\hline \multirow{2}{*}{$\begin{array}{c}\text { Slice } \\
\text { Regis- } \\
\text { ters }\end{array}$} & 15 & 4.5 & 1.8 & 1 \\
\cline { 2 - 5 } & 31 & 6.6 & 2.5 & 1.6 \\
\cline { 2 - 5 } & 63 & 12.8 & 4.4 & 2.7 \\
\hline \multirow{2}{*}{$\begin{array}{c}\text { Slice } \\
\text { LUTs }\end{array}$} & 15 & 23 & 8 & 6 \\
\cline { 2 - 5 } & 31 & 39 & 13 & 9 \\
\cline { 2 - 5 } & 63 & 68 & 26 & 17 \\
\hline
\end{tabular}

The possibilities of implementing the four-position QAM signal demodulator are considered in relation to Cyclone FPGAs produced by Altera. The results of the hardware cost analysis are shown in Table 4.

Table 4: The estimation of the hardware overhead (per cent) of FPGAs produced by Altera for implementing the digital QAM signal demodulator

\begin{tabular}{|c|c|c|c|}
\hline \multirow{2}{*}{ FPGA } & \multicolumn{3}{|c|}{ Elements } \\
\cline { 2 - 4 } & Cyclone IV E & Cyclone V & Cyclone 10 LP \\
& EP4CE22F17 & 5CSEBA6 & 10CL120YF780 \\
& C6 & U23I7DK & C8G \\
\hline Slice & $867 / 22320$ & $763 / 41910$ & $763 / 86800$ \\
Registers & $(3.88 \%)$ & $(1.82 \%)$ & $(1.06 \%)$ \\
\hline Slice & $5274 / 12064$ & $2891 / 22000$ & $2891 / 42400$ \\
LUTs & $(43.26 \%)$ & $(13.14 \%)$ & $(6.81 \%)$ \\
\hline DSP & $4 / 16(25 \%)$ & $8 / 120$ & $8 / 260$ \\
& & $(6.66 \%)$ & $(30.07 \%)$ \\
\hline
\end{tabular}

\section{CONCLUSION}

From the analysis of the obtained results, one can conclude that even relatively inexpensive modern FPGAs allow us to implement various devices for demodulating the digital keyed signals while providing rather acceptable, if not promising, technical features. Unused FPGA resources can be applied to solving other technical tasks by the same equipment.

\section{ACKNOWLEDGMENTS}

This research was financially supported by the Russian Science Foundation (research project No. 17-11-01049).

\section{REFERENCES}

Feher K., 1995. Wireless Digital Communications. Modulation and Spread Spectrum Applications. New Jersey: Prentice-Hall.

Sklar B., 2001. Digital communications: fundamentals and applications. New Jersey: Prentice-Hall.

Proakis J. and Salehi M., 2007. Digital communications. New York: McGraw-Hill.

Chernoyarov O.V., Glushkov A.N., Litvinenko V.P., Litvinenko Yu.V. and Matveev B.V., 2018. Digital demodulator of the quadrature amplitude modulation signals. Measurement Science Review, 18 (6), 236-242.

Glushkov A.N., Litvinenko V.P., Matveev B.V., Chenoyarov O.V. and Kalashnikov K.S., 2016.
Hardware implementation of radio signals fast digital detection and demodulation algorithms. Proceedings of the 2016 International Conference on Communications, Information Management and Network Security (CIMNS2016), pp. 303-306, September 25-26, Shanghai (China).

Chernoyarov O.V., Litvinenko V.P., Glushkov A.N., Matveev B.V. and Salnikova A.V., 2017. Digital demodulation of the signals phase-shift keyed in toto and coded by Walsh sequences. Proceedings of IEEE International Conference on Power, Control, Signals \& Instrumentation Engineering (ICPCSI), pp. 1-5. September 21-22, Chennai (India).

Glushkov A.N., Litvinenko V.P., Matveev B.V., Chernoyarov O.V. and Salnikova A.V., 2015. Basic algorithm for the coherent digital processing of the radio signals. Proceeding of the 2015 International Conference on Space Science \& Communication (IconSpace), pp. 389-392. August 10-12, Langkawi (Malaysia).

Chernoyarov O.V., Glushkov A.N., Litvinenko V.P., Litvinenko Yu.V. and Matveev B.V., 2017. Fast digital algorithms for the coherent demodulation of the phase-shift keyed signals. Proceedings of 2017 IEEE Dynamics of Systems, Mechanisms and Machines (Dynamics), pp. 12-16. November 14-16, Omsk (Russia).

Chernoyarov O.V., Glushkov A.N., Litvinenko V.P., Litvinenko Yu.V. and Matveev B.V., 2018. Fast digital algorithms for the non-coherent demodulation of the differential phase-shift keyed binary signals. International Review of Electrical Engineering, 13 (4), 334-341.

Andina J.J.R., de la Torre Arnanz E. and Valdes M.D., 2017. FPGAs: Fundamentals, advanced features, and applications in industrial electronics. 1st ed. Boca Raton: CRC Press.

Xilinx DS160 Spartan-6 family overview, 2011. San Jose: Xilinx Inc.

7 Series FPGAs data sheet: Overview, 2018. San Jose: Xilinx Inc.

Parab J.S., Gad R.S. and Naik G.M., 2018. Hands-on experience with Altera FPGA development boards. Delhi: Springer India.

\section{AUTHORS BIOGRAPHY}

Oleg V. Chernoyarov was born in 1973. He received his M.Sc. and Ph.D. degrees in radio physics from the Voronezh State University, Voronezh, Russia, and the D.Sc. degree in radio physics - from the Moscow Pedagogical State University, Moscow, Russia, in 1996, 2000 and 2011, respectively. Presently, he is working as a Professor at the Department of Electronics and Nanoelectronics of the National Research University "Moscow Power Engineering Institute" as well as a Leading Research Worker of the National Research Tomsk State University (International Laboratory of Statistics of Stochastic Processes and Quantitative Finance) and the Maikop State Technological 
University (Department of Higher Mathematics and System Analysis). His main interests are statistical methods for transmission and processing of information.

Alexey N. Glushkov was born in 1975 . He received his $\mathrm{Ph} . \mathrm{D}$. in radio engineering from the Voronezh Institute of the Ministry of Internal Affairs of the Russian Federation in 2006. Alexey N. Glushkov is currently working as an Associate Professor at the Department of Infocommunication Systems and Technologies of the Voronezh Institute of the Ministry of Internal Affairs of the Russian Federation. His research interests include digital signal processing, as well as development, modeling and hardware implementation of the algorithms for the synchronization of the digital communication signals and their parameters estimation.

Vladimir P. Litvinenko was born in 1949. He received his Ph.D. in radio enginnering from the All-Union Extra-Mural Electrical Institute of Communications, Moscow, Russia, in 1979. Presently, Vladimir P. Litvinenko is working as an Associate Professor at the Department of Radio Engineering of the Voronezh State Technical University. His main interests are statistical radio physics and radio engineering, digital signal processing.

Alexander N. Faulgaber was born in 1993. He received the B.Sc. and the M.Sc. degrees from the National Research University "Moscow Power Engineering Institute" in 2014 and 2016, respectively, both in radio engineering. Presently, Alexander N. Faulgaber is a $\mathrm{PhD}$ student in the Department of Electronics and Nanoelectronics at the National Research University "Moscow Power Engineering Institute". His research interests include synthesis, analysis and simulation of the processing algorithms of stochastic processes and fields with unknown parameters.

Alexandra V. Salnikova was born in 1978, graduated in geological and hydrogeological engineering from the Voronezh State University in 2000. Presently, Alexandra V. Salnikova is working as an Assistant Professor at the Department of Management Systems and Information Technologies in Building of the Voronezh State Technical University as well as a Researcher of the National Research Tomsk State University (International Laboratory of Statistics of Stochastic Processes and Quantitative Finance) and the National Research University "Moscow Power Engineering Institute" (RD Department "Scientific Production Center "Design Engineering Bureau of Radio Engineering Devices and Systems"). Her research interests include synthesis, analysis and simulation of the detection and estimation algorithms of stochastic signals and fields with unknown parameters. 